\title{
Factors Associated with Streptococcus pneumoniae Nasopharyngeal Carriage and Antimicrobial Susceptibility among Children Under the Age of 5 Years in the Southwestern Colombia
}

\author{
Gustavo Gámez ${ }^{1,20}$ Juan Pablo Rojas ${ }^{3,4,5}$ Santiago Cardona ${ }^{2}$ Juan David Castillo Noreña ${ }^{30}$ \\ María Alejandra Palacio ${ }^{1}$ Luis Fernando Mejía ${ }^{3,4}$ José Luis Torres ${ }^{1}$ Jaime Contreras ${ }^{3}$ \\ Laura Mery Muñoz ${ }^{1}$ Javier Criales ${ }^{3,4}$ Luis Felipe Vélez ${ }^{2}$ Angélica María Forero ${ }^{3,4}$ \\ Yulieth Alexandra Zúñiga ${ }^{2}$ María Eugenia Cuastumal ${ }^{3,4}$ Leidy Johanna Acevedo ${ }^{1}$ \\ Álvaro de Jesús Molina ${ }^{3,4}$ Johan Alexis Bolivar ${ }^{2}$ Alejandro Gómez-Mejia ${ }^{6,7}$ Jessica Lorena Morales ${ }^{1,2}$ \\ Sven Hammerschmidt ${ }^{7}$ \\ ${ }^{1}$ Basic and Applied Microbiology Research Group, School of \\ Microbiology, University of Antioquia, UdeA, Medellín, Colombia \\ ${ }^{2}$ Genetics, Regeneration and Cancer Research Group, University \\ Research Center, University of Antioquia, UdeA, Medellín, Colombia \\ ${ }^{3}$ Club Noel Children's Clinical Foundation, Cali, Colombia \\ 4 Pediatrics Graduate Program, School of Medicine, University Libre \\ Sectional Cali, Cali, Colombia \\ ${ }^{5}$ School of Health, Valle University, Cali, Colombia \\ J Pediatr Infect Dis 2021;16:205-215.

\begin{abstract}
Address for correspondence Gustavo Gámez, School of Microbiology, Universidad de Antioquia, UdeA, Calle 70 \# 52 - 21, 050010 Medellín, Colombia (e-mail: gustavo.gamez@udea.edu.co).

${ }^{6}$ Department of Infectious Diseases and Hospital Epidemiology, University Hospital Zurich, University of Zurich, Zurich, Switzerland

7 Department of Molecular Genetics and Infection Biology, Center for Functional Genomics of Microbes, Interfaculty Institute for Genetics and Functional Genomics, University of Greifswald, Greifswald, Germany
\end{abstract}

Abstract
Keywords
- Streptococcus
pneumoniae
- pneumococcal
children colonization
- nasopharyngeal
carriage
- Colombia

Objective This work aimed to evaluate the factors associated with Streptococcus pneumoniae nasopharyngeal colonization and antimicrobial susceptibility among pediatric outpatients in southwestern Colombia, 2019.

Methods A cross-sectional study was performed using survey-based interviews and the collection of nasopharyngeal-swab specimens for microbiological characterization and antimicrobial susceptibility testing. Logistic regression analyses were performed for factors associated with nasopharyngeal carriage.

Results A total of 452 children under the age of 5 years were examined in which $41.8 \%$ carried S. pneumoniae. Higher pneumococcal carriage frequencies were observed among participants aged $<2$ years and in individuals belonging to indigenous communities, which were lacking established pneumococcal-conjugated vaccine-10 immunization schemes. Additionally, children attending childcare institutions were also highly colonized by pneumococci. S. pneumoniae showed $57.7 \%$ nonsusceptibility to benzyl-penicillin (meningitis-cut); $45.5 \%$ intermediate-sensitivity to benzyl- received

October 31, 2020 accepted after revision

May 1, 2021

published online

June 26, 2021
DOI https://doi.org/ 10.1055/s-0041-1731343. ISSN 1305-7707.
(C) 2021. The Author(s).

This is an open access article published by Thieme under the terms of the Creative Commons Attribution-NonDerivative-NonCommercial-License, permitting copying and reproduction so long as the original work is given appropriate credit. Contents may not be used for commercial purposes, or adapted, remixed, transformed or built upon. (https://creativecommons.org/ licenses/by-nc-nd/4.0/)

Georg Thieme Verlag KG, Rüdigerstraße 14, 70469 Stuttgart, Germany 
penicillin (oral-cut) and $21.7 \%$ to cefotaxime; and resistance to erythromycin (40.7\%), tetracycline (36.0\%), trimethoprim/sulfamethoxazole (24.9\%), clindamycin (24.3\%), and ceftriaxone (27.0\%).

Conclusion The $41.8 \%$ of participants carrying S. pneumoniae show a scenario with the presence of multidrug and extensively drug-resistant strains, which constitutes important reservoirs of bacterial transmission by children aged $<5$ years in Colombia, leading to an onset of pneumococcal diseases. Hence, there is an urgent need to expand conjugate pneumococcal immunization in the community and ensure compliance with established immunization schedules.

\section{Introduction}

Streptococcus pneumoniae is Gram-positive bacteria present in the nasopharyngeal microbiota of healthy humans. Although pneumococcal carriage is usually asymptomatic, it can serve as a reservoir for infections in children, the elderly, immunocompromised people, and individuals with underlying diseases. As a severe pathogen, S. pneumoniae is able to cause community-acquired pneumonia, bacteremia, sepsis, meningitis, otitis media, and sinusitis. ${ }^{1-3} S$. pneumoniae is the leading cause of lower respiratory tract infections worldwide, contributing to more deaths than all other etiologies of lower respiratory tract infection combined. ${ }^{4,5}$ Therefore, it is considered a silent killer of children under the age of 5 years. ${ }^{6}$ Nearly 1 million children under the age of 5 years die each year due to diseases caused by pneumococcus. ${ }^{2}$ In Colombia, pneumonia is one of the main causes of mortality with 13 cases per 100,000 deaths, with S. pneumoniae being its main etiological agent (mortality rate of $3 \%$ ). ${ }^{7}$ Likewise, the average incidence of pneumococcus in Colombia is 0.28 cases per 100,000 inhabitants, with a lethality between 13 and $27 \%$, even with the appropriate treatment of affected individuals., 8

The asymptomatic carriage of $S$. pneumoniae has been identified as a prerequisite for the development of invasive and noninvasive diseases, with carriers being the main source of transmission to other individuals in the community and within hospitals. ${ }^{10,11}$ Several clinical and demographic characteristics, such as infancy, overcrowding, childcare assistance, family size, sibling numbers, poverty, smoking, and recent use of antibiotics, have been associated with pneumococcal colonization. ${ }^{12}$ Although nasopharyngeal isolates are not useful for predicting the causative agent of invasive disease in individuals, they do reflect the epidemiological aspects of diseases caused by $S$. pneumoniae in the community. ${ }^{13,14}$ Bacteria inhabiting the upper respiratory tract of healthy children reflect the strains causing infection that are currently circulating in the community. ${ }^{15}$ Studies in the recent decades have gradually revealed the connection between pneumococcal carriage and infections caused by this pathobiont. ${ }^{10,11}$

For many years, antibiotics such as penicillin and chloramphenicol have been used for the treatment of pneumococcal disease. Unfortunately, the dramatic increase in resistance to antimicrobial agents worldwide has made the choice of antimicrobial drugs for $S$. pneumoniae infections increasingly difficult and expensive. ${ }^{16}$ Currently, prevention campaigns against pneumococcal infections are performed using pneumococcal-conjugated vaccines (PCVs) using those specific serotypes most frequently associated with invasive pneumococcal diseases (IPDs). ${ }^{1,17,18}$ In Colombia, the PCV-7 vaccine was introduced to the National Immunization Program in 2006 and replaced in 2011 by the 10 -valent vaccine $(2+1$ doses $)$.

The decrease in several primary risk factors, the implementation of better immunization strategies, and advances in the treatment of pneumococcal infections have made a substantial progress in recent years in reducing the burden of pneumococcal diseases. However, this is not equally applied worldwide and more research and intervention efforts are still needed. In addition, nasopharyngeal colonization by antibiotic-resistant S. pneumoniae has been steadily increasing, representing potential dangers for the community. ${ }^{4,19}$ In Colombia, and particularly in its southwestern (SW) region (Departments of Valle del Cauca, Cauca, Putumayo and Nariño) (-Fig. 1), epidemiological data on S. pneumoniae are extremely limited. Therefore, the objectives of this study were to evaluate the factors associated with $S$. pneumoniae nasopharyngeal carriage and antimicrobial susceptibility among pediatric outpatients in the SW Colombia, 2019, by determining the frequency of nasopharyngeal colonization and the antimicrobial resistance/susceptibility profile of the pneumococcal isolates. This information will be useful for the implementation of more rational therapeutic and preventive strategies against pneumococcus in Colombia.

\section{Materials and Methods}

A cross-sectional study was conducted in the SW Colombia in 2019 (-Fig. 1). A total of 452 children under the age of 5 years were randomly selected and prospectively involved. For logistical convenience, all eligible participants were those attending the Club Noel Children's Clinical Foundation (CNCCF) for pediatric control. ${ }^{20,21}$ The CNCCF is a secondlevel pediatric hospital that operates every day of the year, serving up to 300 patients per day. Although the population attending the CNCCF comes mainly from the city of Cali, another large number of pediatric patients come from other nearby departments and municipalities.

The sample size of the study population (452 children) was estimated using the general formula for a proportion of a single population with the following assumptions: (1) a total 


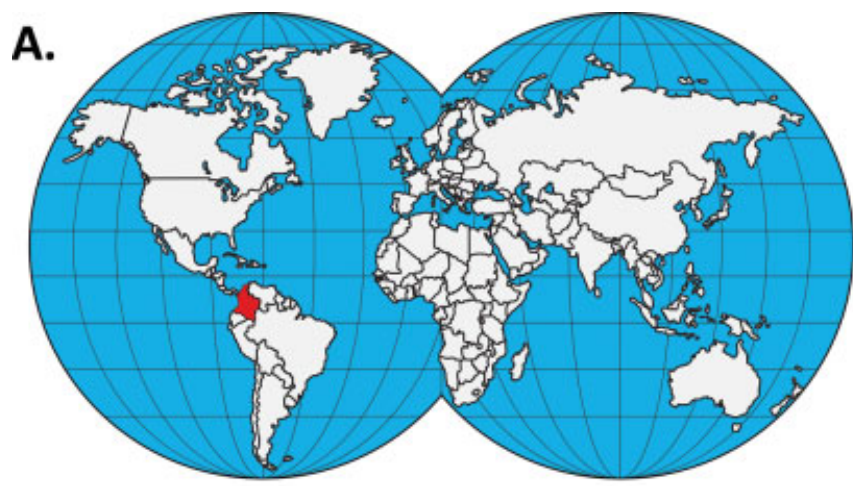

B.

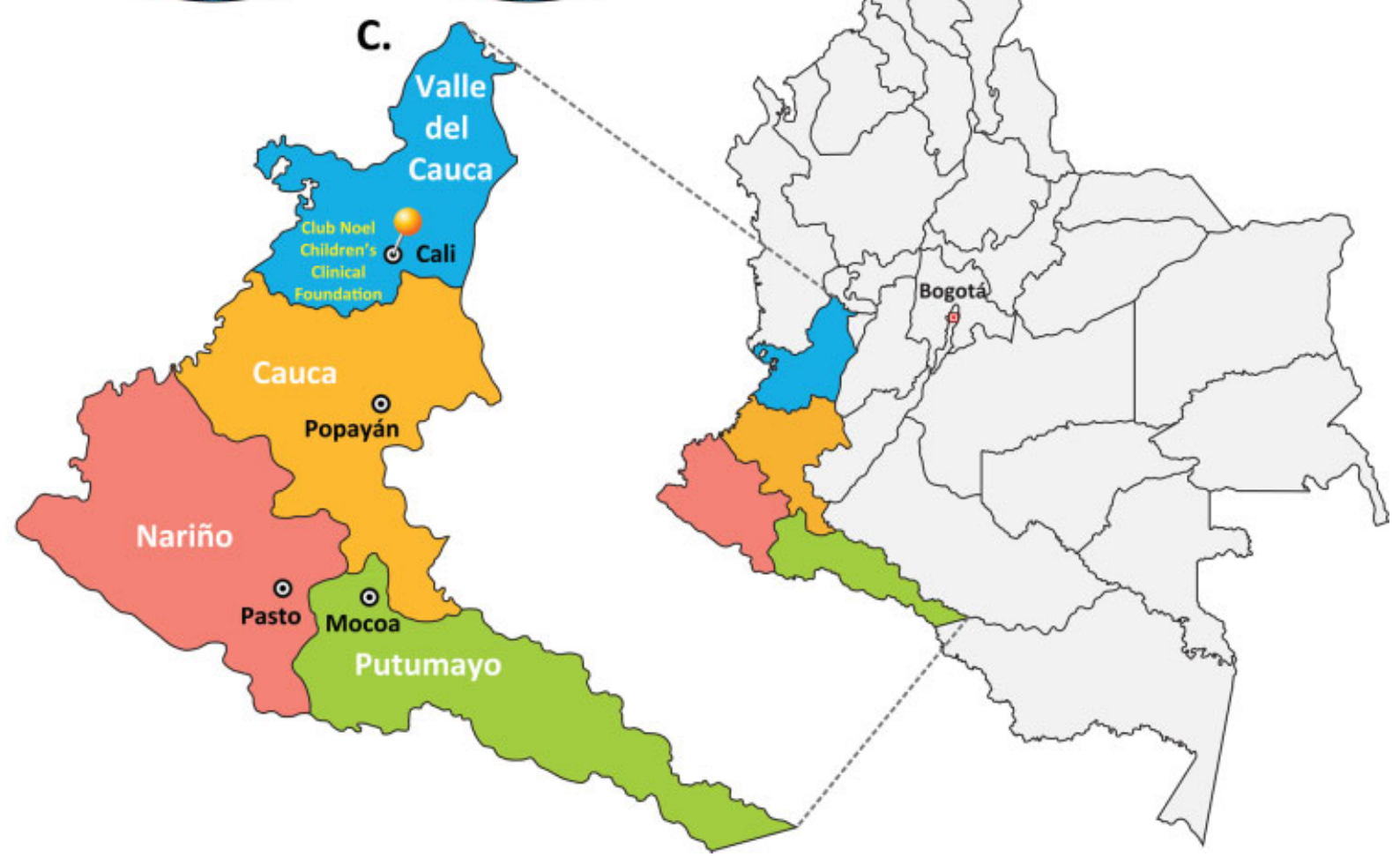

Fig. 1 Geographic location of southwestern Colombia in the World. (A) Republic of Colombia in the world. (B) Southwestern region in the Colombian territory. (C) Departments of Valle del Cauca, Cauca, Nariño and Putumayo and their capitals Cali, Popayán, Pasto, and Mocoa, respectively. Club Noel Children's Clinical Foundation is located in Cali (Valle del Cauca). Bogotá is the capital of Colombia.

population of children under the age of 5 years in the SW Colombia of $734,372^{22}$; (2) a prevalence rate of pneumococcal nasopharyngeal colonization of 50\%; (3) A 95\% confidence level (CI); and (4) a $20 \%$ of marginal error or possible loss of information. The main exclusion criteria were: (1) children under the age of 5 years with IPD or any other acute, moderate, or severe illness; (2) children who had received antibiotics in the previous month or who had previously received any immunosuppressive medication; (3) those children whose parents or guardians were unwilling or unable to give their informed consent freely and spontaneously; and (4) those who did not accomplished the inclusion criteria were also excluded.

This study was approved by the Bioethics Committee of the CNCCF. Informed consents were signed by parents or guardians of each child for voluntary participation acceptance. Each participant was anonymized by a code to keep confidential the information. Sociodemographic and housing data of the participants were collected through a standardized and previously tested survey, applied to the parents or guardians of each child. Likewise, data were collected on the clinical history of the participating children such as use of antibiotics and other medications, the history of the diseases suffered, including severe diseases such as anemia, cerebral paralysis and hip dysplasia, among others. PCV-10 immunization records were also collected and grouped into the following categories: not immunized, incomplete immunization, immunization in process (for children under 1 ), and complete immunization (for children over-1) (-Tables 1 and 2).

Nasopharyngeal swab specimens were collected from each child using sterile flexible swabs (Copan, Brescia, Italy), according to standardized procedures. ${ }^{23}$ Each collected swab was immediately introduced into a cryovial containing STGG transport medium (skimmed milk solution-tryptone-glucose-glycerol), ${ }^{23}$ for preservation at $-30^{\circ} \mathrm{C}$ in the 
Factors Associated with Streptococcus pneumoniae Nasopharyngeal Carriage and Antimicrobial

Table 1 Baseline characteristics and their association with Streptococcus pneumoniae nasopharyngeal colonization in 452 outpatient children of the Club Noel Children's Clinic, involved in the study, in 2019

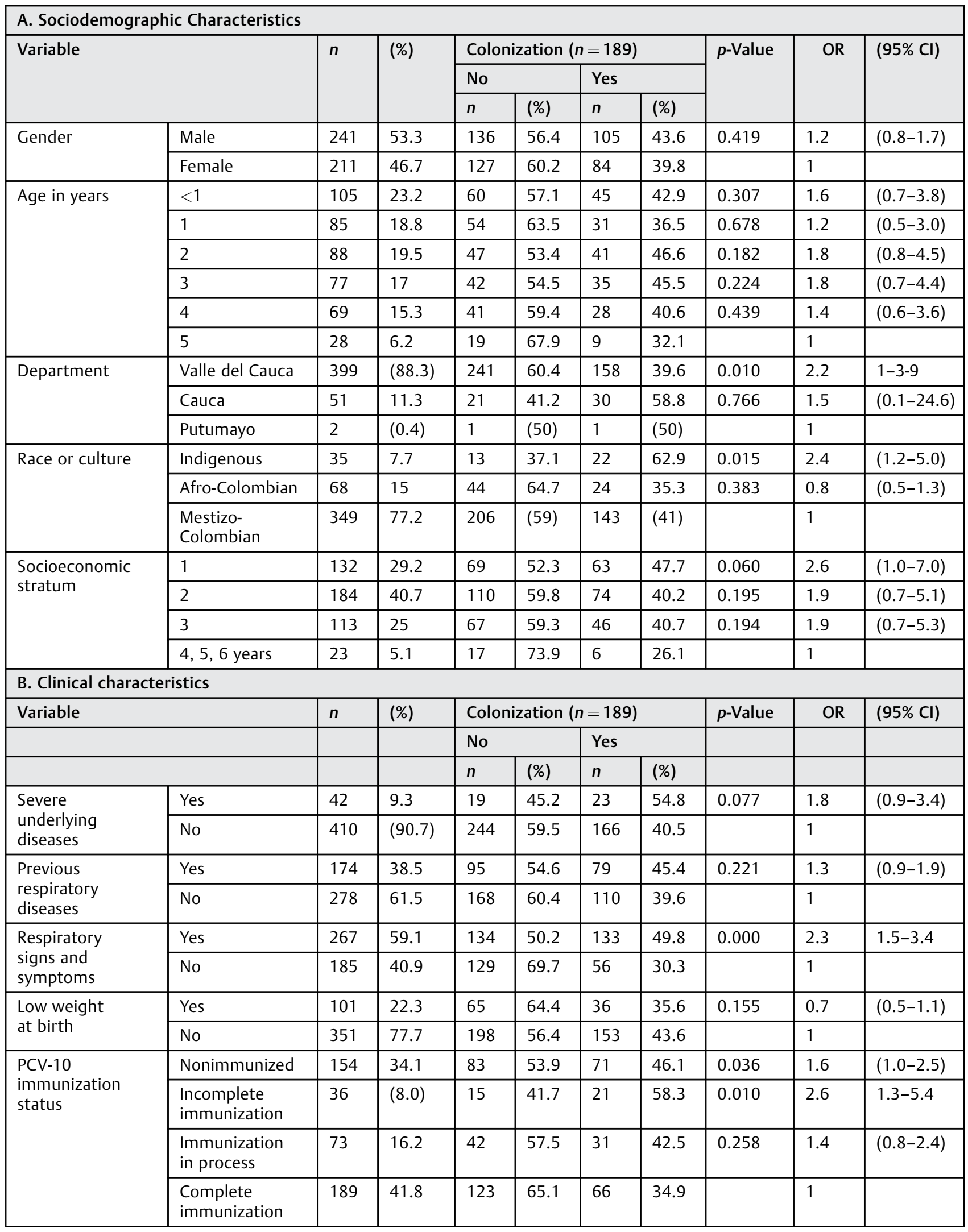


Table 1 (Continued)

\section{Characteristics of the living quarters, home, and life habits}

\begin{tabular}{|c|c|c|c|c|c|c|c|c|c|c|}
\hline \multicolumn{2}{|l|}{ Variable } & \multirow[t]{3}{*}{$n$} & (\%) & \multicolumn{4}{|c|}{ Colonization $(n=189)$} & \multirow[t]{3}{*}{ p-Value } & \multirow[t]{3}{*}{ OR } & \multirow[t]{3}{*}{$(95 \% \mathrm{Cl})$} \\
\hline & & & \multicolumn{2}{|l|}{ No } & \multicolumn{2}{|l|}{ Yes } & & & & \\
\hline & & & $n$ & (\%) & $n$ & (\%) & & & & \\
\hline \multirow[t]{2}{*}{ Overcrowding } & Yes & 51 & 11.3 & 27 & 52.9 & 24 & 47.1 & 0.421 & 1.3 & $(0.7-2.3)$ \\
\hline & No & 401 & $(88.7)$ & 236 & 58.9 & 165 & 41.1 & & 1 & \\
\hline \multirow[t]{2}{*}{ Shared bedroom } & Yes & 354 & 78.3 & 206 & 58.2 & 148 & 41.8 & 0.996 & 1.0 & $(0.6-1.6)$ \\
\hline & No & 98 & 21.7 & 57 & 58.2 & 41 & 41.8 & & 1 & \\
\hline \multirow{2}{*}{$\begin{array}{l}\text { Living with } \\
\text { smoker(s) }\end{array}$} & Yes & 61 & 13.5 & 38 & 62.3 & 23 & 37.7 & 0.485 & 0.8 & $(0.5-1.4)$ \\
\hline & No & 391 & $(86.5)$ & 225 & 57.5 & 166 & 42.5 & & 1 & \\
\hline \multirow{2}{*}{$\begin{array}{l}\text { Contact with } \\
\text { sick person(s) }\end{array}$} & Yes & 47 & 10.4 & 30 & 63.8 & 17 & 36.2 & 0.408 & 0.8 & $(0.4-1.4)$ \\
\hline & No & 405 & $(89.6)$ & 233 & 57.5 & 172 & 42.5 & & 1 & \\
\hline \multirow{2}{*}{$\begin{array}{l}\text { Hospitalized } \\
\text { home member(s) }\end{array}$} & Yes & 27 & 6 & 14 & 51.9 & 13 & 48.1 & 0.492 & 1.3 & $(0.6-2.9)$ \\
\hline & No & 425 & (94) & 249 & 58.6 & 176 & 41.4 & & 1 & \\
\hline \multirow{2}{*}{$\begin{array}{l}\text { Child care center } \\
\text { attendance }\end{array}$} & Yes & 178 & 39.4 & 91 & 51.1 & 87 & 48.9 & 0.014 & 1.6 & $(1.1-2.4)$ \\
\hline & No & 274 & 60.6 & 172 & 62.8 & 102 & 37.2 & & 1 & \\
\hline
\end{tabular}

Abbreviations: AOR, adjusted odd ratios; $95 \% \mathrm{Cl}$, 95\% confidence interval; PCV-10, 10-valent pneumococcal conjugate vaccine. $n=452$; $p$-value $=0.05$.

Note: Not immunized means no vaccination at all, complete immunization is when children aged over 1 year have completed the scheme $2+1$, immunization in process is only for children under the age of 1 years and means to have received the number of doses for the age in months, and incomplete immunization means any other situation where the number of doses and/or booster does not match with the age.

Microbiology Laboratory of CNCCF. Samples collected each week were transported in dry ice to the Central Research Laboratory of the School of Microbiology of the University of Antioquia in Medellín, Colombia.

For the culture and isolation of the pneumococcus, an aliquot of the sample was used for growth in tryptic soy agar solid medium, supplemented with defibrinated sheep blood (5\%), yeast extract $(0.5 \%)$, and gentamicin $(5 \mu \mathrm{g} / \mathrm{mL})$. Pneumococci were incubated at $37^{\circ} \mathrm{C}$ and $5 \% \mathrm{CO}_{2}$ for a maximum of 24 hours, after which the following tests and assays were necessary to confirm their identification: (1) evidence of $\alpha$-Hemolysis; (2) Gram staining; (3) optochin sensitivity test, isolates with an inhibition zone $\geq 14 \mathrm{~mm}$ in diameter were considered susceptible to optochin; and (4) bile solubility test. Finally, stocks were generated for the storage of the isolates, through the use of Todd-Hewitt supplemented with yeast extract liquid culture medium, supplemented with glycerol in cryovials, which were then preserved in an ultra-freezer at $-80^{\circ} \mathrm{C}^{2,23}$

To test the susceptibility/resistance of the colonizing isolates against different antibiotics, fresh pneumococcal cultures were made on Mueller-Hinton Agar plates, supplemented with Ram's Blood (5\%) and incubated for 14 hours at $37^{\circ} \mathrm{C}$ and $5 \% \mathrm{CO}_{2}$. The inoculum was then prepared in sterile $0.45 \%$ saline solution by resuspending the colonies until a turbid suspension equivalent to a 0.5 McFarland standard. ${ }^{24}$ Subsequently, the identification tests (GP Test Cards for Gram-positive cocci) and antimicrobial sensitivity (AST03 Cards for Streptococcal Susceptibility) were performed, using the VITEK-2 system from BioMérieux, according to the manufacturer's instructions. The AST03 susceptibility cards contain Wilkins-Chalgren culture medium, modified with the following antimicrobial agents: benzyl-penicillin (meningitis, oral, and pneumonia), ceftriaxone (meningitis and other), cefotaxime (meningitis and other), vancomycin, erythromycin, tetracycline, clindamycin, chloramphenicol, linezolid, tigecycline, trimethoprim/sulfamethoxazole, levofloxacin, moxifloxacin, and rifampicin. The pneumococcal strain ATCC 49619 was used as a control. According to the CLSI criteria, colonizing pneumococcal isolates were classified as sensitive, sensitive intermediate or resistant, according to established cutoff points. ${ }^{2,24}$

Data were tabulated, validated, and analyzed using the Excel program and the Statistical Package for Social Sciences (SPSS) version 20.0 (IBM Corporation, Chicago, Illinois, United States). The statistical analysis was performed as follows: (1) descriptive statistics were used to summarize the sociodemographic information, the frequency of nasopharyngeal carriage, the molecular characteristics, and the antimicrobial resistance/susceptibility of the isolates; (2) multivariate regression analysis (in which the enter method was applied) was performed to identify possible factors associated with the nasopharyngeal carriage of pneumococci (adjusted odds ratio [AOR]; 95\% CI). Fisher's exact test and chi-squared test of independence were performed before to identify the candidate variables for the multivariate analysis, according to the HosmerLemeshow Criteria, cut-point $p<0.25$ (OR: crude odds ratio; $95 \% \mathrm{CI}$ ). $p$-Values $<0.05$ were considered statistically significant. 
Factors Associated with Streptococcus pneumoniae Nasopharyngeal Carriage and Antimicrobial

Table 2 Multivariate analysis of associated factors for Streptococcus pneumoniae nasopharyngeal colonization in 452 outpatient children of the Club Noel Children's Clinic, involved in the study, in 2019

\begin{tabular}{|c|c|c|c|c|}
\hline Variable & p-Value & AOR & $(95 \% \mathrm{Cl})$ & \\
\hline \multirow[t]{2}{*}{ Gender } & Male & 0.554 & 1.1 & $(0.7-1.7)$ \\
\hline & Female & & 1 & \\
\hline \multirow[t]{6}{*}{ Age in years } & $<1$ & 0.115 & 2.8 & $(0.8-10.2)$ \\
\hline & 1 & 0.173 & 2.1 & $(0.7-6.4)$ \\
\hline & 2 & 0.031 & 3.1 & $(1.1-8.9)$ \\
\hline & 3 & 0.068 & 2.6 & $(0.9-7.1)$ \\
\hline & 4 & 0.201 & 1.9 & $(0.7-5.4)$ \\
\hline & 5 & & 1 & \\
\hline \multirow[t]{3}{*}{ Race or culture } & Indigenous & 0.035 & 2.4 & $(1.1-5.4)$ \\
\hline & Afro-Colombian & 0.348 & 0.7 & $(0.4-1.4)$ \\
\hline & Mestizo-Colombian & & 1 & \\
\hline \multirow[t]{4}{*}{ Socioeconomic stratum } & 1 & 0.157 & 2.2 & $(0.7-6.3)$ \\
\hline & 2 & 0.365 & 1.6 & $(0.6-4.6)$ \\
\hline & 3 & 0.382 & 1.6 & $(0.6-4.7)$ \\
\hline & $4,5,6$ years & & 0.0 & \\
\hline \multirow[t]{2}{*}{ Severe underlying disease (total) } & Yes & 0.207 & 1.6 & $(0.8-3.3)$ \\
\hline & No & & 1 & \\
\hline \multirow[t]{2}{*}{ Previous respiratory disease (asthma) } & Yes & 0.117 & 1.7 & $(0.9-3.4)$ \\
\hline & No & & 1 & \\
\hline \multirow[t]{2}{*}{ Previous respiratory disease (rhinitis) } & Yes & 0.180 & 1.9 & $(0.8-4.6)$ \\
\hline & No & & 1 & \\
\hline \multirow[t]{2}{*}{ Respiratory signs and symptoms (nasal secretion) } & Yes & 0.003 & 2.1 & $(1.3-3.4)$ \\
\hline & No & & 1 & \\
\hline \multirow[t]{2}{*}{ Respiratory signs and symptoms (sneezing) } & Yes & 0.230 & 0.7 & $(0.4-1.2)$ \\
\hline & No & & 1 & \\
\hline \multirow[t]{2}{*}{ Respiratory signs and symptoms (cough) } & Yes & 0.102 & 1.6 & $(0.9-2.7)$ \\
\hline & No & & 1 & \\
\hline \multirow[t]{2}{*}{ Respiratory signs and symptoms (phlegm) } & Yes & 0.808 & 1.1 & $(0.6-2.0)$ \\
\hline & No & & 1 & \\
\hline \multirow[t]{4}{*}{ PCV-10 immunization status } & Nonimmunized & 0.049 & 1.7 & $(1.0-2.7)$ \\
\hline & Incomplete immunization & 0.007 & 3.0 & $(1.3-6.7)$ \\
\hline & Immunization in process & 0.256 & 1.8 & $(0.6-5.1)$ \\
\hline & Complete immunization & & 1 & \\
\hline \multirow[t]{2}{*}{ Overcrowding } & Yes & 0.395 & 1.3 & $(0.7-2.6)$ \\
\hline & No & & 1 & \\
\hline \multirow[t]{2}{*}{ Child care center attendance } & Yes & 0.039 & 1.8 & $(1.0-3.2)$ \\
\hline & No & & 1 & \\
\hline
\end{tabular}

Abbreviations: AOR, adjusted odd ratios; 95\% Cl, 95\% confidence interval; PCV-10, 10-valent pneumococcal conjugate vaccine. $n=452 ; p$ value $=0.05$; Not immunized means no vaccination at all, complete immunization is when children aged over 1 year have accomplished the scheme $2+1$, immunization in process is only for children under the age of 1 year and means to have received the number of doses for the age in months, and incomplete immunization means any other situation where the number of doses and/or booster does not match with the age. 


\section{Results}

A total of 452 individuals under the age of 5 years from SW Colombia, who attended the CNCCF for outpatient services between September and October of 2019, were included in this study. From this population, 241 (53.3\%) were male and 211 (46.7\%) were female. With regard to age, 23.2\% (105) children were less than 1 year old, $18.8 \%$ (85) were 1 year old, $19.5 \%$ (88) children were 2 years old, $17.0 \%$ (77) were 3 years old, $15.3 \%$ (69) 4 years old, and $6.2 \%^{25}$ children were 5 years old. The average age of the children was 30 months with a standard deviation of 18 months. The median age of the children was 28.5 months and the interquartile range was 31 months. Regarding race or ethnicity, 349 (77.2\%) identified themselves as mestizo-Colombians, 68 (15.1\%) AfroColombians, and 35 (7.7\%) indigenous people belonging to Amerindian communities settled mainly in the Department of Cauca. The majority of children $94.5 \%$ (429) belonged to socioeconomic strata 1,2 , and 3 (low/medium-income levels), while only two participants belonged to stratum 6 (high-income level) from the municipalities of Yumbo and Jamundí. Moreover, 399 (88.3\%) participating children came from 21 different municipalities in the Department of Valle del Cauca of which 244 (61.1\%) lived in the city of Cali. Participation of 51 (11.3\%) children was obtained from the Department of Cauca, while from the Department of Nariño no children could be included/involved during the sampling period (-Table 1A).

Forty-two (9.3\%) participating children were clinically diagnosed with severe diseases such as anemia, cerebral paralysis, and hip dysplasia, among others. At some point in their life, 174 (38.5\%) children were diagnosed with a respiratory illness such as pneumonia, asthma, bronchitis, otitis, and rhinitis, among others. However, they were fully recovered at the time of sampling. Furthermore, 267 (59.1\%) participants were diagnosed with respiratory signs and symptoms at the time the samples were collected, such as nasal secretion, cough, sneezing, and phlegm, among others. Regarding the immunization status, 298 (65.9\%) participants had received at least one dose of the PCV-10. Only 187 (41.4\%) participants certified to have a complete PCV-10 immunization schedule $(2+1$ doses) (-Table 1B).

Regarding the conditions of housing, home, and life habits, $51(11.3 \%)$ children participating in the study lived in overcrowded conditions ( 3 or more people per bedroom), while 98 (21.7\%) children had their own room and slept without companions in their own bed. Finally, 61 (13.5\%) participants lived with people who smoke cigarettes regularly, while 178 (39.4\%) of the children attended childcare institutions near their homes (-Table 1C).

Of the 452 children examined, 189 (41.8\%) were carriers of $S$. pneumoniae. The highest frequency of pneumococcal nasopharyngeal colonization was observed in 2-year-old children (41 children, 46.6\%). The overall frequency of $S$. pneumoniae nasopharyngeal carriage was $43.6 \%$ in males versus $39.8 \%$ in females. Children belonging to Indigenous communities had the highest frequency of nasopharyngeal colonization (62.9\%), while the lowest frequency was ob- served in Afro-Colombian participants (35.3\%). In children with the lowest socio-economic condition (Stratum 1), the overall frequency of nasopharyngeal carriage of S. pneumoniae was $47.7 \%$. Although the number of children from higher socio-economical groups was low (23 participants out of 452 ), the frequency of pneumococcal colonization was much lower $(26.1 \%)$. The colonization frequency of children from the Department of Cauca was 58.8\% (- Table 1A).

Having been clinically diagnosed with a severe disease (54.8\%), respiratory disease at any time in life (45.4\%), and respiratory signs and symptoms at the time of sampling (49.8\%) were the variables identified with higher frequencies of nasopharyngeal colonization by $S$. pneumoniae, when compared with the healthy children group. Likewise, the colonization frequencies of children who were not immunized $(71,46.1 \%)$ or with incomplete PCV-10 immunization schedules (47.7\%) were higher than those children with complete PCV-10 immunization schedule (34.8\%) (-Table 1B, -Fig. 1).

The frequency of $S$. pneumoniae nasopharyngeal colonization of participants attending child care institutions in the vicinity of their homes (48.9\%) was higher than that of participants not attending any institution (37.2\%). Similarly, the frequency of nasopharyngeal colonization was $47.1 \%$ among children living in overcrowded conditions versus $41.8 \%$ in children who do not share their room and sleep alone in their bed. Conversely, the frequency of colonization of participants living with people smoking regularly at home (37.7\%) was lower than that of participants living with nonsmokers (42.5\%) (-Table 1C).

The results showed a risk correlation between pneumococcal colonization and the 2-year age group (AOR $=3.1 ; 95 \%$ $\mathrm{CI}=1.1-8.9 ; p=0.031)$. The nasopharyngeal carriage of $S$. pneumoniae was significantly higher in children belonging to indigenous communities $(\mathrm{AOR}=2.4 ; 95 \% \quad \mathrm{CI}=1.1-5.4$; $p=0.035$ ). In addition, there was a significant risk association between nasopharyngeal colonization of $S$. pneumoniae and nasal secretion at the time of sampling $(A O R=2.1 ; 95 \%$ $\mathrm{CI}=1.3-3.4 ; p=0.003)$. Failure to complete PCV-10 immunization schedules $(\mathrm{AOR}=3.0 ; 95 \% \mathrm{CI}=1.3-6.7 ; p=0.007)$ and not having received immunization against pneumococcus (AOR $=1.7 ; 95 \% \mathrm{CI}=1.0-2.7 ; p=0.049$ ) were significantly associated with the presence of $S$. pneumoniae in the nasopharynx in SW Colombia (-Fig. 2). Likewise, attending childcare institutions $(\mathrm{AOR}=1.8 ; 95 \% \mathrm{CI}=1.0-3.2 ; p=0.039)$ was significantly correlated with pneumococcal nasopharyngeal colonization. However, there was no significant association between gender, socioeconomic stratum, severe diseases, respiratory disease at any time in life (asthma, pneumonia, rhinitis, etc.), respiratory signs and symptoms at the time of sample collection (cough, sneezing and phlegm, among others), low weight at birth, and overcrowding with pneumococcal nasopharyngeal carriage ( - Table 2 ).

The resistance/susceptibility profiles of the colonizing isolates to 18 antibiotics contained in the AST-03 card (VITEK-2) are reported in - Table 3. Fifty-five (29.1\%) pneumococcal colonizing isolates were susceptible to all antibiotics tested, 31 (16.4\%) were resistant to an antimicrobial 


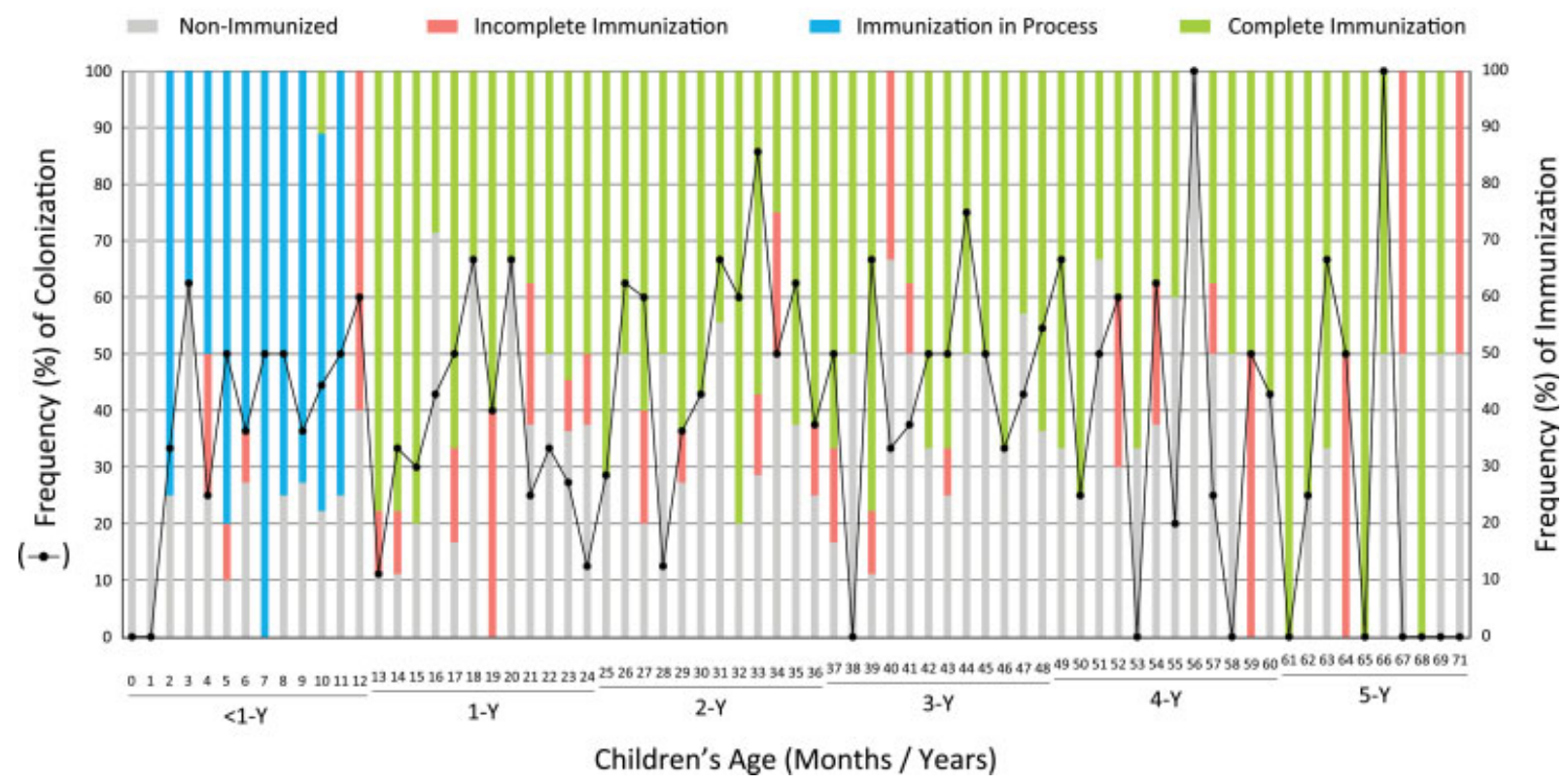

Fig. 2 Frequency of nasopharyngeal colonization by Streptococcus pneumoniae and 10-valent pneumococcal conjugate vaccine (PCV-10) immunization status according to the age in months (and years) of participating children. Southwestern Colombia, 2019.

agent, and 26 (13.8\%) were resistant to two. Sixty-nine (36.5\%) colonizing isolates were resistant to between three and ten different antibiotics (multidrug resistant [MDR]), while 23 (12.2\%) presented resistance profiles to at least one antibiotic in each class of antimicrobial agents (extensivelydrug resistant [XDR]). Moreover, 109 (57.7\%) pneumococcal clinical isolates not susceptible to benzyl-penicillin (cut meningitis) were identified and 86 (45.5\%) presented a reduced or intermediate susceptibility to this antibiotic, according to the oral cutoff threshold. Seventy-seven (40.7\%) colonizing isolates were resistant to erythromycin, 68 (36.0\%) to tetracycline, 47 (24.9\%) to trimethoprim/sulfamethoxazole, and $46(24.3 \%)$ to clindamycin. Regarding ceftriaxone and cefotaxime (third-generation cephalosporins), 51 (27.0\%) and $41(21.7 \%)$ pneumococcal isolates were identified with resistance profiles and intermediate susceptibility, respectively.

\section{Discussion}

In this study, a frequency of pneumococcal nasopharyngeal carriage of $41.8 \%$ among children under the age of 5 years was observed for the first time in SW Colombia. The risk factors found to be mainly associated with pneumococcal nasopharyngeal colonization are children who are 2 years of age (46.6\%) and children attending childcare institutions (48.9\%). Interestingly, the age of 2 years matches perfectly with the times when parents begin their children's schooling in this region of the country. However, in children who were 3, 4, and 5 years of age, a nonsignificant decrease in the frequency of pneumococcal colonization was observed, which could be a reflection of the gradual acquisition of mucosal immunity from the upper respiratory tract, especially in children with complete PCV-10 immunization schemes against pneumococcus, and reduced exposure to the pathogen in nurseries and their homes. These results are in agreement with the nasopharyngeal colonization frequencies observed in other countries worldwide. They are of great relevance because exposure to other children during childhood, especially peers in community care institutions, has been clearly associated with an increased risk of colonization and IPD and non-IPD. ${ }^{14,20,21,26,27}$

A significant finding of our study is the fact that children belonging to indigenous communities are those that are most vulnerable to the nasopharyngeal presence of $S$. pneumoniae (62.9\%). These indigenous children are from lower income levels (socioeconomic strata 1 and 2) and have lower vaccine uptake (60\%). This further contributed to a colonization frequency above the average for the Department of Cauca (58.8\%). These results coincide with reports from other Latin American countries with similar conditions to those observed in this Colombian region. ${ }^{14,25,28-30}$ However, the number of participants belonging to the indigenous communities of the departments Cauca and Nariño was low, due to the geographic distances to Cali. This emerged as an important limitation of our study.

In addition to age and ethnicity, the logistic regression analysis was associated with pneumococcal colonization frequencies with nasal secretion at the time of sampling and with a lack or incomplete PCV-10 immunization schemes $(2+1)$ (-Fig. 2$)^{3,11}$ These results are similar to those found in reports from countries with similar health conditions. $^{31-33}$

The results of the susceptibility study revealed higher antibiotic resistance of $S$. pneumoniae to relatively cheap and 
Table 3 Antimicrobial susceptibility profiles of Streptococcus pneumoniae colonizing strain, isolates of the 452 patients involved in the study

\begin{tabular}{|c|c|c|c|c|c|c|}
\hline \multirow[t]{3}{*}{ Antimicrobial agent } & \multicolumn{6}{|c|}{ S. pneumoniae strains $(n=189)$} \\
\hline & \multicolumn{2}{|c|}{ Resistant } & \multicolumn{2}{|c|}{ Intermediate } & \multicolumn{2}{|c|}{ Susceptible } \\
\hline & $n$ & $(\%)$ & $n$ & (\%) & $n$ & $(\%)$ \\
\hline Benzyl-penicillin (meningitis) & 109 & 57.7 & 0 & $(0)$ & 80 & 42.3 \\
\hline Erythromycin & 77 & 40.7 & 0 & (0) & 112 & 59.3 \\
\hline Tetracycline & 68 & $\begin{array}{l}(36.0) \\
\end{array}$ & 0 & $(0)$ & 121 & $(64.0)$ \\
\hline Trimethoprim/sulfamethoxazole & 47 & 24.9 & 15 & 7.9 & 127 & 67.2 \\
\hline Clindamycin & 46 & 24.3 & 5 & 2.6 & 138 & $(73.0)$ \\
\hline Benzyl-penicillin (oral) & 23 & 12.2 & 86 & 45.5 & 80 & 42.3 \\
\hline Ceftriaxone (meningitis) & 17 & $(9.0)$ & 14 & 7.4 & 158 & $\begin{array}{l}(83.6) \\
\end{array}$ \\
\hline Cefotaxime (meningitis) & 15 & 7.9 & 14 & 7.4 & 160 & $(84.7)$ \\
\hline Ceftriaxone (another) & 10 & 5.3 & 7 & 3.7 & 172 & $\begin{array}{l}(91.0) \\
\end{array}$ \\
\hline Cefotaxime (Another) & 9 & 4.8 & 6 & 3.2 & 174 & $\begin{array}{l}(92.1) \\
\end{array}$ \\
\hline Benzyl-penicillin (pneumonia) & 4 & 2.1 & 5 & 2.6 & 180 & $(95.2)$ \\
\hline Vancomycin & 0 & (0) & 0 & (0) & 189 & $(100)$ \\
\hline Chloramphenicol & 0 & $(0)$ & 0 & $(0)$ & 189 & $(100)$ \\
\hline Linezolid & 0 & (0) & 0 & (0) & 189 & $(100)$ \\
\hline Tigecycline & 0 & (0) & 0 & (0) & 189 & $(100)$ \\
\hline Levofloxacin & 0 & $(0)$ & 0 & $(0)$ & 189 & (100) \\
\hline Moxifloxacin & 0 & (0) & 0 & (0) & 189 & $(100)$ \\
\hline Rifampicin & 0 & (0) & 0 & $(0)$ & 189 & $(100)$ \\
\hline
\end{tabular}

readily available antibiotics for the population such as benzylpenicillin cut meningitis (57.7\%), erythromycin (40.7\%), tetracycline (36.0\%), and trimethoprim/sulfamethoxazole (24.9\%), and more expensive but of variable use alternatives such as ceftriaxone (27.0\%) and clindamycin (24.3\%). This observation is consistent with previous reports in Venezuela and other countries in the world. ${ }^{19,34-36}$ On the other hand, vancomycin, chloramphenicol, linezolid, tigecycline, levofloxacin, moxifloxacin, and rifampicin were the most effective antibiotics against S. pneumoniae isolates, all with $100 \%$ antimicrobial susceptibility, which is consistent with reports from other countries of the region. ${ }^{19,37}$ Twenty-three (12.2\%) colonizing isolates presented resistance profiles to at least one antibiotic in each class of antimicrobial agents, which include them in the dangerous XDR group. Likewise, 69 (36.5\%) isolates were resistant to between three and ten different antibiotics, being considered in this study as MDR. Less than one-third of the pneumococci isolated in SW Colombia were susceptible to all antibiotics tested, which is a direct product of frequent and inappropriate use of chemotherapeutics. Although data on the use of different antibiotics in low- and middle-income countries are underrepresented, the pneumococcal resistance frequency to antimicrobial agents varies according to geographic region and the different population subgroups analyzed. ${ }^{16,38}$ These variations represent major challenges for health systems in Latin American countries and reflect the uncontrolled and low-cost availability of some of these medical resources. ${ }^{16}$ This phenomenon is not foreign to Colombia and its regions, ${ }^{9,39,40}$ which would be exerting greater selection pressure for resistant $S$. pneumoniae strains, favoring the increase in their frequency and, therefore, decreasing the efficacy of these antibiotics in the treatment of $S$. pneumoniae affected patients.

In conclusion, a general frequency of $S$. pneumoniae nasopharyngeal colonization of $41.8 \%$ is reported for SW Colombia, and our findings reveal that 2 years old children, attending childcare centers, are at a higher risk of acquiring $S$. pneumoniae and suffering from their diseases. Moreover, indigenous communities, presenting nasal secretion, and not being immunized against the pneumococcus or not completing the established PCV-10 immunization schemes, were identified as risk factors for nasopharyngeal carriage of S. pneumoniae. On the other hand, a nonsusceptibility of $S$. pneumoniae to benzyl-penicillin (meningitis and oral cuts), increased resistance to antibiotics erythromycin, tetracycline, trimethoprim/sulfamethoxazole, and clindamycin was observed, in addition to resistance and intermediate levels of susceptibility to cephalosporin of broad spectrum (ceftriaxone and cefotaxime).

This study presents for the first time the regional frequency data of children under the age of 5 years carrying $S$. pneumoniae in SW Colombia. This high frequency of children carrying S. pneumoniae could show an important reservoir of bacterial transmission among children in the community, 
which could potentially lead to the onset of pneumococcal diseases with serious consequences for the health of people in this Colombian region. Therefore, there is a clear need to expand pneumococcal conjugate immunization in the community and ensure compliance with established PCV-10 immunization schedules. Finally, the demonstration of the association of nasopharyngeal colonization of resistant MDR and XDR-like strains with the development of invasive infection by resistant strains is important to establish rational treatments for the alleged S. pneumoniae infections in SW Colombia.

\section{Authors' Contributions}

G.G., J.P.R., L.F.M., J.C.O., L.J.A., J.A.B., A.G.M., J.L.M., and S.H. were involved in conceptualization. G.G., J.P.R., S.C., M.A.P., J.L.T., L.M.M., L.F.V., Y.A.Z., and J.L.M. were involved in data curation. G.G., J.P.R., J.L.M., and S.H. were involved in formal analysis. G.G. assisted in funding acquisition. All authors were involved in investigation and in methodology. G.G. was involved in project administration and managed resources. G.G., J.P.R., and S.H. were involved in supervision. G.G. and J.L.M. were involved in validation. G.G. and J.L.M. were involved in visualization. G.G, A.G.M., and J.L.M. were involved in writing of original draft. G.G., J.P.R., S.C., J.D.C., M.A.P., L.F.M., J.L.T., J.C.O., L.M.M., J.C.R., L.F.V., A.M.F., Y.A.Z., M.E.C., L.J.A., A.J.M., J.A.B., A.G.M., J.L.M., and S.H. were involved in writing and editing of the draft.

\section{Funding}

This work was supported by Pfizer, Inc., through the grant: IIR WI244770.

\section{Conflict of Interest}

G.G. reports grants from Pfizer, Inc, during the conduct of the study. Rest authors declare no conflict of interest.

\section{Acknowledgments}

The authors thank Jaime Dominguez Navia, Luz Myriam Claros, María Victoria Hernández, María Victoria Muñoz, María del Palmar, Jhonny Castrillón, and all the staff of Club Noel Children's Clinical Foundation, Cali, Colombia for facilitating the realization of this study.

\section{References}

1 Brown J, Hammerschmidt S, Orihuela C. 2015Streptococcus pneumoniae: Molecular Mechanisms of Host-Pathogen Interactions. ELSEVIER. Accessed May 20, 2021 at: https://www.elsevier.com/ books/streptococcuspneumoniae/brown/978-0-12-410530-0

2 WHO. 2003 Manual for the laboratory identification and antimicrobial susceptibility testing of bacterial pathogens of public health concern in the developing world. Accessed May 20, 2021 at: https://www.who.int/csr/resources/publications/drugresist/WHO_CDS_CSR_RMD_2003_6/en/

3 Walker CLF, Rudan I, Liu L, et al. Global burden of childhood pneumonia and diarrhoea. Lancet 2013;381(9875):1405-1416

4 GBD 2016 Lower Respiratory Infections Collaborators. Estimates of the global, regional, and national morbidity, mortality, and aetiologies of lower respiratory infections in 195 countries, 19902016: a systematic analysis for the Global Burden of Disease Study 2016. Lancet Infect Dis 2018;18(11):1191-1210
5 O'Brien KL, Wolfson LJ, Watt JP, et al; Hib and Pneumococcal Global Burden of Disease Study Team. Burden of disease caused by Streptococcus pneumoniae in children younger than 5 years: global estimates. Lancet 2009;374(9693):893-902

6 Rodríguez L, Cervantes E, Ortiz R. Malnutrition and gastrointestinal and respiratory infections in children: a public health problem. Int J Environ Res Public Health 2011;8(04):11741205

7 Martínez-Vernaza S, Mckinley E, Soto MJ, Gualtero S. Community acquired pneumonia: a narrative review. Univ Med 2018;59(04): 93-102

8 Castañeda-Orjuela C, Alvis-Guzmán N, de la Hoz-Restrepo F. Impacto de la enfermedad por Streptococcus pneumoniae en población adulta mayor en Bogotá, Colombia, 2008. Rev Salud Públ 2010;12(01):38-50

9 Salud INd. Publicaciones Informes y boletines de vigilancia por laboratorio. Informe Nacional de SIREVA II - Colombia 20062018. SIREVA II 2019:16. Accessed May 20, 2021 at: http://www. ins.gov.co/buscador-eventos/Paginas/Informes-y-boletines-devigilancia-por-laboratorio-micro.aspx\#InplviewHash6c8e35e266db-4a75-8f06-fb3c6e13971f=WebPartID\%3D\%7B6C8E35E266DB-4A75-8F06-FB3C6E13971F\%7D-FilterField1\%3DLaboratorio-FilterValue1 \%3DMicrobiolog\%25C3\%25ADa

10 Bogaert D, De Groot R, Hermans PWM. Streptococcus pneumoniae colonisation: the key to pneumococcal disease. Lancet Infect Dis 2004;4(03):144-154

11 Simell B, Auranen K, Käyhty H, Goldblatt D, Dagan R, O’Brien KLPneumococcal Carriage Group. The fundamental link between pneumococcal carriage and disease. Expert Rev Vaccines 2012; 11(07):841-855

12 Bogaert D, van Belkum A, Sluijter M, et al. Colonisation by Streptococcus pneumoniae and Staphylococcus aureus in healthy children. Lancet 2004;363(9424):1871-1872

13 Brueggemann AB, Griffiths DT, Meats E, Peto T, Crook DW, Spratt BG. Clonal relationships between invasive and carriage Streptococcus pneumoniae and serotype- and clone-specific differences in invasive disease potential. J Infect Dis 2003;187(09): 1424-1432

14 Mackenzie GA, Leach AJ, Carapetis JR, Fisher J, Morris PS. Epidemiology of nasopharyngeal carriage of respiratory bacterial pathogens in children and adults: cross-sectional surveys in a population with high rates of pneumococcal disease. BMC Infect Dis 2010;10:304

15 Faden H, Duffy L, Wasielewski R, Wolf J, Krystofik D, Tung Y Relationship between nasopharyngeal colonization and the development of otitis media in children. Tonawanda/Williamsville Pediatrics. J Infect Dis 1997;175(06):1440-1445

16 Zanichelli V, Monnier AA, Gyssens IC, et al. Variation in antibiotic use among and within different settings: a systematic review. J Antimicrob Chemother 2018;73(06, Suppl 6):vi17-vi29

17 Gamez G, Hammerschmidt S. Combat pneumococcal infections: adhesins as candidates for protein-based vaccine development. Curr Drug Targets 2012;13(03):323-337

18 WHO. Pneumococcal Vaccines World Health Organization Position Paper. Wkly Epidemiol Rec 2003;78:97-120. Accessed May 20, 2021 at: http://www.who.int/immunization/policy/position_papers/pneumococcus/en/

19 Quintero B, Araque M. [Serotype profile and antibiotyping of Streptococcus pneumoniae strains isolated from nasal carriage in pediatric patients]. Invest Clin 2006;47(01):17-26

20 Assefa A, Gelaw B, Shiferaw Y, Tigabu Z. Nasopharyngeal carriage and antimicrobial susceptibility pattern of Streptococcus pneumoniae among pediatric outpatients at Gondar University Hospital, North West Ethiopia. Pediatr Neonatol 2013;54(05):315-321

21 Hernandez-Bou S, Garcia-Garcia JJ, Gene A, Esteva C, del Amo E, Muñoz-Almagro C. Pneumococcal carriage in children attending a hospital outpatient clinic in the era of pneumococcal conjugate 
vaccines in Barcelona. Diagn Microbiol Infect Dis 2012;74(03): 258-262

22 DANE. Colombia. 2018. Demografía y población. Accessed May 20, 2021 at: https://www.dane.gov.co/index.php/estadisticas-portema/demografia-y-poblacion

23 Satzke C, Turner P, Virolainen-Julkunen A, et al; WHO Pneumococcal Carriage Working Group. Standard method for detecting upper respiratory carriage of Streptococcus pneumoniae: updated recommendations from the World Health Organization Pneumococcal Carriage Working Group. Vaccine 2013;32(01):165-179

24 CLSI. Performance Standards for Antimicrobial Susceptibility Testing. 29th edition. CLSI supplement M100 Wayne, PA: Clinical and Laboratory Standards Institute; 2019

25 Otsuka T, Chang B, Shirai T, et al; SADO-study Working Group. Individual risk factors associated with nasopharyngeal colonization with Streptococcus pneumoniae and Haemophilus influenzae: a Japanese birth cohort study. Pediatr Infect Dis J 2013;32(07):709-714

26 Ercibengoa M, Arostegi N, Marimón JM, Alonso M, Pérez-Trallero E. Dynamics of pneumococcal nasopharyngeal carriage in healthy children attending a day care center in northern Spain. Influence of detection techniques on the results. BMC Infect Dis 2012;12:69

27 Navne JE, Børresen ML, Slotved HC, et al. Nasopharyngeal bacterial carriage in young children in Greenland: a population at high risk of respiratory infections. Epidemiol Infect 2016;144(15): 3226-3236

28 Neves FPG, Pinto TCA, Corrêa MA, et al. Nasopharyngeal carriage, serotype distribution and antimicrobial resistance of Streptococcus pneumoniae among children from Brazil before the introduction of the 10-valent conjugate vaccine. BMC Infect Dis 2013;13:318

29 Usuf E, Bottomley C, Adegbola RA, Hall A. Pneumococcal carriage in sub-Saharan Africa-a systematic review. PLoS One 2014;9(01): e85001

30 Vallès X, Flannery B, Roca A, et al. Serotype distribution and antibiotic susceptibility of invasive and nasopharyngeal isolates of Streptococcus pneumoniae among children in rural Mozambique. Trop Med Int Health 2006;11(03):358-366

31 Espinosa-de Los Monteros LE, Jiménez-Rojas V, Aguilar-Ituarte F, et al. Streptococcus pneumoniae isolates in healthy children attending day-care centers in 12 states in Mexico. Salud Publica Mex 2007;49(04):249-255
32 Masuda K, Masuda R, Nishi J, Tokuda K, Yoshinaga M, Miyata K. Incidences of nasopharyngeal colonization of respiratory bacterial pathogens in Japanese children attending day-care centers. Pediatr Int 2002;44(04):376-380

33 Zemlicková H, Urbásková P, Adámková V, Motlová J, Lebedová V, Procházka B. Characteristics of Streptococcus pneumoniae, Haemophilus influenzae, Moraxella catarrhalis and Staphylococcus aureus isolated from the nasopharynx of healthy children attending day-care centres in the Czech Republic. Epidemiol Infect 2006; 134(06):1179-1187

34 Birindwa AM, Emgård M, Nordén R, et al. High rate of antibiotic resistance among pneumococci carried by healthy children in the eastern part of the Democratic Republic of the Congo. BMC Pediatr [Internet] Accessed May 20, 2021 on: https://www.ncbi.nlm.nih. gov/pmc/articles/PMC6241069/

35 Emgård M, Msuya SE, Nyombi BM, et al. Carriage of penicillinnon-susceptible pneumococci among children in northern Tanzania in the 13-valent pneumococcal vaccine era. Int J Infect Dis 2019;81:156-166

36 Stacevičienė I, Petraitienė S, Vaičiūnienė D, Alasevičius T, Kirslienè J, Usonis V. Antibiotic resistance of Streptococcus pneumoniae, isolated from nasopharynx of preschool children with acute respiratory tract infection in Lithuania. BMC Infect Dis 2016;16 (01):216

37 Gazi H, Kurutepe S, Sürücüoğlu S, Teker A, Ozbakkaloglŭ B Antimicrobial susceptibility of bacterial pathogens in the oropharynx of healthy school children in Turkey. Indian J Med Res 2004;120(05):489-494

38 Bayer M, Aslan G, Emekdaş G, Kuyucu N, Kanik A. [Nasopharyngeal carriage of Streptococcus pneumoniae in healthy children and multidrug resistance]. Mikrobiyol Bul 2008;42(02): 223-230

39 José Pallares C, Martínez E. Implementación de un programa de uso regulado de antibióticos en 2 unidades de cuidado intensivo medico-quirúrgico en un hospital universitario de tercer nivel en Colombia. Infectio 2012;16(04):192-198

40 Leal AL, Castañeda E. [Antimicrobial susceptibility of Streptococcus pneumoniae colonizing the nasopharynx of Colombian children with pneumonia]. Rev Panam Salud Publica 1997;1(04): 266-272 